\title{
TRITA-EPP-90-03
}

Potential Drops Supported by Ion Density

Cavities in the Dynamic Response of a

Plasma Diode to an Applied Field

M. Bohm and S. Torvén

June 1990

Department of Plasma Physics The Royal Institute of Technology

S-10044 Stockholm Sweden 
Potential Drops Supported by Ion Density Cavities in the Dynamic Response of a Plasma Diode to an Applied Field

M. Bohm and S. Torvén

Department of Plasma Physics, Royal Institute of Technology, S-100 44 Stockholm

Abstract

Experiments have shown that an applied voltage drop may either be supported by a cathode sheath or by a quasi-linear variation over the plasma lasting for several electron transit times. In the latter case an ion density cavity existed initially. An analytical model and numerical simulations are used to show that a cavity gives rise to a quasi-linear potential variation for applied voltage drops below a certain critical value. For larger values the drop concentrates to a cathode sheath. The quasi-linear profile steepens to a double layer for large cavity depths. 
Introduction

Electric double layers ( $D L$ ) have been studied extensively in low density plasmas, but the significant mechanisms in different processes for DL formation are still unclear. In experiments, where ionization of the background gas by electron impacts is suppressed in the experimental chamiver, the experimental results may seem to be ambigous. When a voltage drop was applied between the two hot plates in a $Q$-machine, operated in the "electron rich regime", most of the the applied drop first concentrated to a cathode sheath. On the time scale of the subsequent ion motion the sheath detached from the cathode and moved into the plasma as a DL [1] [2]. In a similar experiment the response of a weakly magnetized argon plasma column in a differentially pumped triple plasma machine was investigated. The sources were connected to the plasma column by circular apertures where potential drops of the order of $k_{B} T_{e} / e$ reflected some of the source electrons back to the sources like the sheaths in an electron rich $Q$-machine. Most of the applied voltage drop first appeared to be quasi-linearly distributed along the plasma column, and the electron emission from the low potential source was limited by a potential minimum. A DL formed on the time scale of the ion motion by a steepening of this profile [3]. The time resolution of the potential measurements was closely equal to the electron transit time between the sources in the two experiments. In the initial state the density of the plasma column in the triple plasma machine varied axially due to radial ion losses, and for symmetric operation the plasma column formed a symmetric ion density cavity. Ion density cavities have been shown to be of importance in the theory of strong turbulence, and it has been shown by numerical simulations with periodic boundary conditions that cavities generated by a low frequency wave can support small applied potential drops [4]. These results were used to interprete electric fields observed above the Aurora [5] [6]. Ion 
density cavities can also strongly modify the non-linear evolution of plasma oscillations [7]. In this paper we shall present an analytical model demonstrating that a density cavity can support very large potential drops and compare the potential profiles evaluated theoretically with profiles simulated numerically.

\section{Plasma simulations}

In contrast to the earlier simulations [4] we here present simulations of a cavity in an electron rich plasma diode for comparison with the experiments. A one-dimensional "particle in cell" code [8] is used. The time step was $\left(2 \omega_{\mathrm{pe}}\right)^{-1}$, the grid size $\lambda_{D} / 4$, the unit of length $\lambda_{D}=$ $\left(k_{B} T_{e} / m_{e}\right)^{1 / 2} / \omega_{p e}$, and the unit of time $\omega_{p e}{ }^{-1}$ where $\omega_{p e}=\left(N_{1} e^{2} / m_{e} \varepsilon_{0}\right)^{1 / 2}$ and $N_{1}$ is defined below. The maximum density was $10^{3}$ particles per Debye length. The electric potential $\phi(x)$ and the applied potential drop $\phi_{a}$ are measured in units of $k_{B} T_{e} / e$. lons and electrons are injected symmetrically at the two boundaries with half-Maxwellian velocity distributions with a density $N_{0}$. We use the natural ion to electron mass ratio (argon), and the simulations cover a few electron transit times (less than $300 \omega_{\mathrm{pe}}{ }^{-1}$ ). For such short simulation times the velocity distribution of the ions present initially is of little significance as was shown by several runs with different velocity distributions. Here we assume that the initial velocity distributions are Maxwellian with the same temperatures as those of the particles injected. The short simulation time also justifies the use of a one-dimensional model to simulate the triple plasma machine experiment. In contrast to the insignificance of the ion motion, the initial ion density distribution $n_{i p}(x)$ is of great importance since it may give rise to an electric field controlling the electron motion. We consider a diode in the region $0<x<L$ with sheath regions in $0<x<d_{1}$ and $L-d_{1}<x<L$ where $d_{1}<L$. As a suitable test profile in $d_{1}<x<L-d_{1}$, we use 


$$
n_{i p}(x)=N_{1}-\Delta N \cos ^{2}\left\{\pi(x-L / 2) / L_{p}\right\} .
$$

and $n_{i p}(x)=N_{1}$ in the sheath regions. $N_{1}$ is the density at the plasmasheath boundaries and $L_{p}=L-2 d_{1}$. The profile describes a symmetric cavity with a relative depth of $\Delta N / N_{1}$. We have also tested the significance of the ion density drops in the sheaths by using the following profile in $0<x<L$.

$$
n_{i}(x)=n_{i p}(x)+\left(2 N_{0}-N_{1}\right)\{2-q(x)\}
$$

Here $q(x)=\tanh (x / d)+\tanh ((L-x) / d)$ and $d \ll d_{1} \ll L$ so that $n_{j}(x)$ reduces approximately to $n_{i p}(x)$ in the plasma region. The profile is symmetric around $L / 2$ and gives $n_{i}(0) \approx n_{i}(L) \approx 2 N_{0}$ which is twice as large as the density $\mathrm{N}_{0}$ of the electrons and ions injected at each boundary. In the initial, symmetric state this gives quasi-neutrality at the boundaries which then simulates the sources in a steady state triple plasma machine. Simulations for $d / \lambda_{D}$ equal to 10,5 , and $d \rightarrow 0$, that is, $n_{i}(x)=N_{1}$ throughout the sheath regions, showed that the exact shape of the ion density profile in the sheath regions is of no significance for the potential profiles obtained in the plasma region. Below we consider the case $d \rightarrow 0$ and $N_{0}>N_{1}$. The initial potential profile was obtained by a presimulation of a short-circuited diode $\left(\phi_{a}=0\right)$ during $0<t<t_{1}$. For $t=0$ we put $n_{e}(x)=n_{j}(x)$ so that the electric potential vanished everywhere. After 20 or 30 plasma periods a self-consistent potential profile was obtained with sheath potential drops approximately given by $\left(k_{B} T_{e} / e\right) \ln \left(2 N_{0} / N_{1}\right)$ as shown in Fig. 1a for $t=19$. For $t=t_{1}$ a potential drop was applied, and a linear potential variation was superimposed on the initial profile. In Fig. 1 a and $1 \mathrm{~b}$ examples are given of the quasilinearly varying profiles obtained. These remained approximately 
stationary during the whole simulation time after an initial transient lasting for about a transit time. Fig.1c shows that the quasi-linear potential profile steepens to a DL for large cavity depths which is a type of DL not yet observed experimentally. When $\phi_{a}$ exceeds a certain critical value, $\phi_{a}=\phi_{a}$, which was found to be a function of only $N_{0} /\left(N_{1}-\right.$ $\Delta N$ ), the quasi-linear potential variation disappears, and the drop concentrates to a cathode sheath (Fig. 1d). The critical voltage drops may be inferred from simulations for different values of $\phi_{a}, \Delta N / N_{1}$, and $N_{0} / N_{1}$. They agree with the theoretical values of $\phi_{a l}$ derived below for $\phi_{a}$ up to about 10 as shown in Fig. 2. For larger values of $\phi_{a}$ the electron flux from the high potential side becomes strongly attenuated due to reflection, when a potential minimum exists, and the particle density in the simulations is too small to describe this situation properly.

\section{Comparison with an analytical model}

To evaluate the electron number density theoretically, we assume that the normalized potential $\phi(x)$ decreases monotonically from the value $\phi_{C}$ at $x=0$ to a minimum at $x=x_{0}$, where we put $\phi=0$ (in the diagrams $\phi-$ $\phi_{c}$ has been plotted), and then increases monotonically to $\phi_{c}+\phi_{a}$ at $x=L$. The steady electron number density can then be expressed as [9]

$$
n(\phi)=n_{0} \exp \left(\phi-\phi_{c}\right)\left(\operatorname{coth}\left(\phi_{a} / 2\right) \pm \operatorname{erf} \sqrt{\phi}\right) .
$$

where $n_{0}=N_{0}\left(1-\exp \left(-\phi_{a}\right)\right)$. The upper sign gives the density $n_{1}(\phi)$ for $x<x_{0}$ and the lower sign $n_{2}(\phi)$ for $x>x_{0}$. The error function is normalized to unity for large values of its argument. Two branches are obtained due to the asymmetry introduced by the potential $\phi_{a}$ applied at $x=L, n_{1}(\phi)$ increases monotonically whereas $n_{2}(\phi)$ decreases from $n(0)$ to a minimum for $\phi=\phi_{\mathrm{m}}$ and increases monotonically for $\phi>\phi_{\mathrm{m}}$. Accordingly this minimum can coincide with the ion density minimum at $x=L / 2$ only 
if $x_{0}<L / 2 . \phi_{m}$ is determined as a monotonically increasing function of $\phi_{a}$ through the relation

$$
\operatorname{coth}\left(\phi_{a} / 2\right)=\operatorname{erf} \sqrt{ } \phi_{m}+\exp \left(-\phi_{m}\right) /\left(\pi \phi_{m}\right)^{1 / 2}
$$

As a first condition for a quasi-neutral state, $\phi_{c}$ must adjust itself through a change of the sheath space charge to give quasi-neutrality at the density minimum. From (1), (3), and (4) $\phi_{c}$ is determined by

$$
N_{1}-\Delta N=n_{0} \exp \left(-\phi_{c}\right) /\left(\pi \phi_{m}\right)^{1 / 2}
$$

It is conveniant to normalize $n_{1}$ and $n_{2}$ to the minimum density given by the right hand side of (5), and use (4) to eliminate $\operatorname{coth}\left(\phi_{a} / 2\right)$. On the assumption that the ion density profile changes insignificantly during the first few electron transit times, we then obtain the quasineutrality condition as

$$
n_{i p}(x) /\left(N_{1}-\Delta N\right)=\exp \left(\phi-\phi_{m}\right)+\left(\pi \phi_{m}\right)^{1 / 2} \exp (\phi)\left(\operatorname{erf} \sqrt{ } \phi_{m} \pm \operatorname{erf} \sqrt{ } \phi\right)
$$

where the right hand side of (6) gives the normalized electron densities $F_{1}$ and $F_{2}$ corresponding to $n_{1}$ and $n_{2} . \phi(x)$ can now be evaluated for given values of $\Delta N / N_{1}$ by specifying a value of $\phi_{m}$ corresponding to any desired value of $\phi_{a}$. Eq. (6) also gives the potential levels $\phi_{1}$ and $\phi_{2}$ at the plasma sheath boundaries. Fig. 3 shows that there is an excellent agreement between the profiles evaluated and those simulated. Also the sheath potential drops, $\phi_{c}-\phi_{1}$ and $\phi_{c}+\phi_{a}-\phi_{2}$ where $\phi_{c}$ is determined by determined by (5), agree with those simulated.

The normalized density $g\left(\phi_{m}\right)$ at the potential minimum, which is given by the right hand side of ( 6 ) for $\phi=0$, is a monotonically increasing 
function of $\phi_{a}$ through (4). When $\phi_{a}$ is increased for a fixed ion density profile, the potential minimum accordingly moves towards the left boundary since $x_{0}<L / 2$. When $x_{0}$ reaches the position $x=d_{1}$, no solutions with a potential minimum in the plasma region exist for larger values of $\phi_{a}$. However, on the assumption that the potential minimum continues to exist in the cathode sheath (as indicated by the simulations), a quasi-neutral solution still exists in the plasma region, $n_{\text {ip }}(x)=\left(N_{1}-\Delta N\right) F_{2}(\phi)$. The electron number density is then given by $\left(N_{1}-\right.$ $\Delta \mathrm{N} \mathrm{F}_{2}(\phi)$ and $\left(\mathrm{N}_{1}-\Delta \mathrm{N}\right) \mathrm{F}_{1}(\phi)$ in the cathode sheath since (3) holds in the whole region $0<x<\mathrm{L}$. When $\phi_{\mathrm{a}}$ is increased further, the potential minimum will accordingly continue to move towards the left boundary, and finally it reaches the boundary $x=0$. In this state the boundary conditions give the electron number density as $N_{0}\left\{1+\exp \left(-\phi_{a}\right)\right\}$ which must be equal to the density at the potential minimum, $\left(N_{1}-\Delta N\right) g\left(\phi_{m}\right)$. The maximum value of $\phi_{a}, \phi_{a l}$ (shown in Fig. 2), that is consistent with a potential minimum in the diode, is accordingly given by

$$
g\left(\phi_{m}\right) /\left(1+\exp \left(-\phi_{a}\right)\right)=N_{0} /\left(N_{1}-\Delta N\right)
$$

A supplementary condition on $N_{0}, N_{0}\left\{1+\exp \left(-\phi_{a l}\right)\right\}>N_{1}$, is obtained from the condition that the electron number density at $x=0$ always must be larger than $N_{1}$. When $\left(\Delta N / N_{1}\right) \rightarrow 1,(7)$ predicts arbitrary large values of $\phi_{a 1}$. Since (7) is based on the assumption of quasi-neutrality at the density minimum, further investigations including the Poisson equation are necessary to explore this limiting case.

\section{Discussion and conclusions}

The good agreement between the potential profiles simulated and those calculated shows that the theoretical approach is appropriate. One basic assumption in the theoretical model is the existence of a 
potential minimum. This introduces the depth of the minimum as a free parameter which makes it possible to adjust the electron number density to maintain quasi-neutrality for the boundary conditions given. The other assumption is that the ion motion can be neglected during the first few electron transit times and that a quasi-stationary state exists on the time scale of the electron transit time. The agreement between theory and simulations shows that both of these assumptions are fulfilled.

As another interesting consequence of the theory we have found a condition for the existence of the potential minimum in the diode and shown that this coincides with the condition for the existence of a quasi-linear potential variation over the plasma. When a minimum exists, all the field lines from the surface charge on the diode plates ends on charges in the plasma, and the field from each of the plates penetrates the plasma only up to the potential minimum. The plates are "screened" from each other. When the position of the potential minimum reaches the cathode plate, the negative charge distributed in the diode exactly compensates for the positive charge on the anode plate. One would expect this to be a limiting steady state since the potential minimum disappears for a further increase of the applied voltage. As shown by the simulations, most of the applied potential drop then concentrates to a cathode sheath.

The quasi-linearly varying profiles are similar to those observed experimentally, but simultaneously measured ion density profiles are required for a detailed comparison. The limit given in Fig. 2 is consistent with the experimental results because $\phi_{a}$ was of the order of 1000 in the $Q$-machine experiment whereas $\phi_{a}$ was typically 10 in the 
triple plasma machine experiment. However, new experiments with accurately known values of $\mathrm{N}_{0} / \mathrm{N}_{1}$ are required to check the critical voltage drops for the transition between the two states observed.

In summary, we have shown that an ion density cavity can support large potential drops in a quasi-linear potential variation with a spatial scaling constant of the order of the cavity width. For large values of the cavity depth and the applied voltage, the potential profile steepens and the potential drop concentrates to a double layer formed close to the density minimum. When the applied voltage drop exceeds a certain critical value, which depends on $N_{0} /\left(N_{1}-\Delta N\right)$, a transition occurs to a state where most of the applied voltage concentrates to a cathode sheath.

\section{Acknowledgements}

This work has been financed by the Swedish Natural Science Research Council. We wish to thank Dr P. Gray for providing us with suitable numerical codes and Dr:s I. Axnäs, N. Brenning, A. Peratt, N. Singh, and M. A. Raadu for useful discussions.

\section{References}

[1] S. lizuka et al., "Dynamics of a potential barrier formed on the tail of a moving double layer in a collisionless plasma," Phys. Rev. Lett., vol. 48, p.145, 1982.

[2] S. lizuka et al., "Double layer dynamics in a collisionless magnetoplasma," J. Phys. Soc. Japan, vol. 54, p. 2516, 1985.

[3] M. Bohm and S. Torvén, "Extended potential drops preceding 
double layer formation in a triple plasma device," XVIII Int. Conf. on Phenomena in Ionized Gases, Swansea, Contributed Papers, vol. 2, p. 318 1987. (Bristol: The Inst. of Physics Publishing Div.).

[4] H. L. Rowland and P. J. Palmadesso, "Spiky parallel dc electric fields above the aurora," J. Geophys. Res., vol. 92, p. 299, 1987.

[5] M. Temerin, K. Cerny, W. Lotko, and F. S. Mozer, "Observations of double layers and solitary waves in the auroral plasma," Phys. Rev. Lett., vol. 48_p.1175, 1982.

[6] R. Boström, G. Gustafsson, B. Holback, G. Holmgren, H. Koskinen, and P. Kintner, "Characteristics of solitary waves and weak double layers in the magnetospheric plasma," Phys. Rev. Lett., vol. 61_p. 82, 1988.

[7] E. Infeld, G. Rowlands, and S. Torvén, "Ion density cavities can cause nonlinear plasma oscillations to peak," Phys. Rev. Lett., vol. 62, p. 2269, 1989.

[8] W. S. Lawson, "Particle simulation of bounded 1D plasma systems," J. of Computational Phys., vol.80, p. 253, 1989.

[9] M. Bohm and S. Torvén, "The dynamic response of an inhomogeneous plasma diode to an applied electric field", to be published 

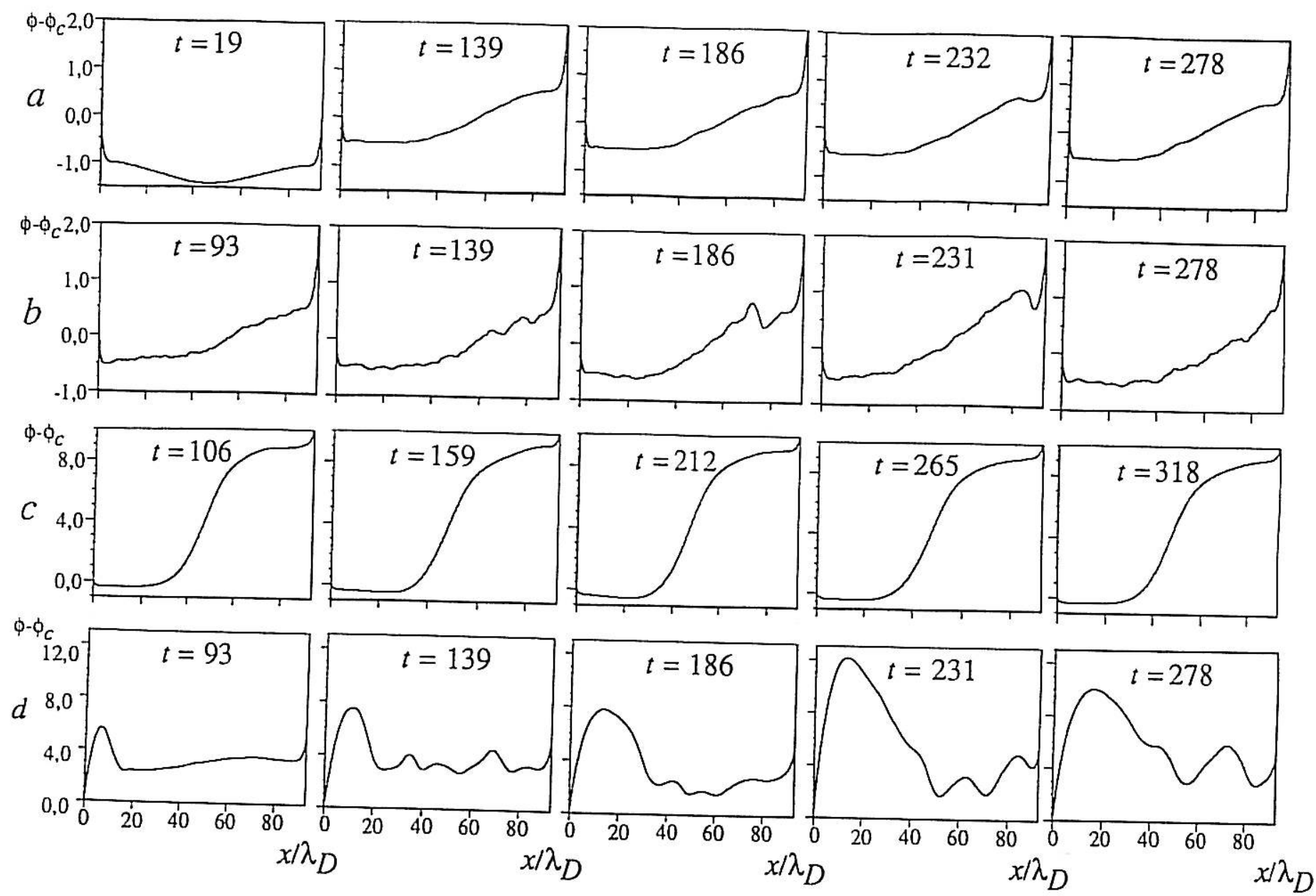

Fig.1. Simulated potential profiles $\phi-\phi_{c}$ at times given in $\omega_{p e}{ }^{-1}$. At the left boundary $\phi-\phi_{c}=0$. A voltage drop $\phi_{a}$ was applied between the boundaries at $t \approx 25$. Fig. 1a shows shows the initial profile $(t=19)$ and profiles at later times which are averaged over $50 \omega_{p e}{ }^{-1}\left(\phi_{a}=2, \Delta N / N_{1}=0.3, N_{0} / N_{1}=1.36\right)$. Fig. 1b shows instantanous profiles for the same parameters. Fig.1c shows the steepening to a $D L$ for large cavity depths $\left(\phi_{a}=10, \Delta N / N_{1}=0.83\right.$, $\left.N_{0} / N_{1}=1.04\right)$. In Fig.1d $\phi_{a}$ exceeds the critical voltage drop shown in Fig. 2 $\left(\phi_{a}=5, \Delta N / N_{1}=0.3, N_{0} / N_{1}=1.36\right)$. The profiles in $1 \mathrm{c}$ and $1 \mathrm{~d}$ are averages over $10 \omega_{\mathrm{pe}}{ }^{-1}$. 


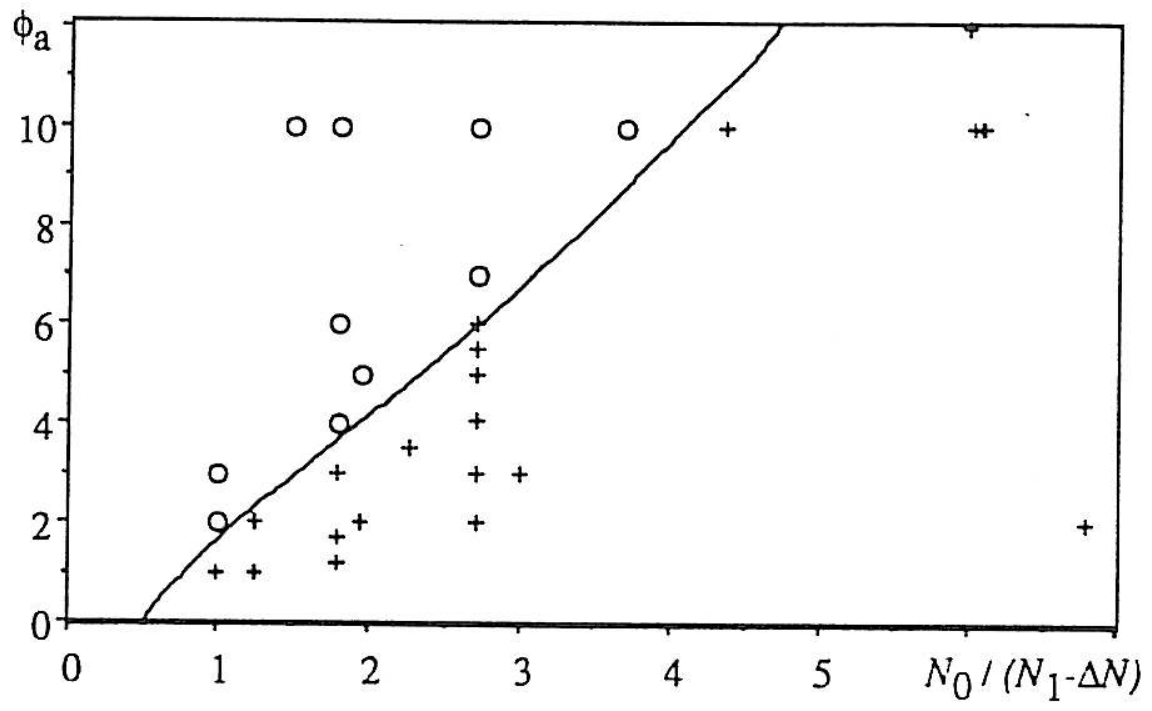

Fig. 2. The circles denote simulations giving voltage drops concentrated to a cathode sheath and the crosses those giving quasi-linear potential variations with a potential minimum. The full line gives the maximum value of the applied voltage consistent with the existence of a potential minimum according to (7) and (4). 


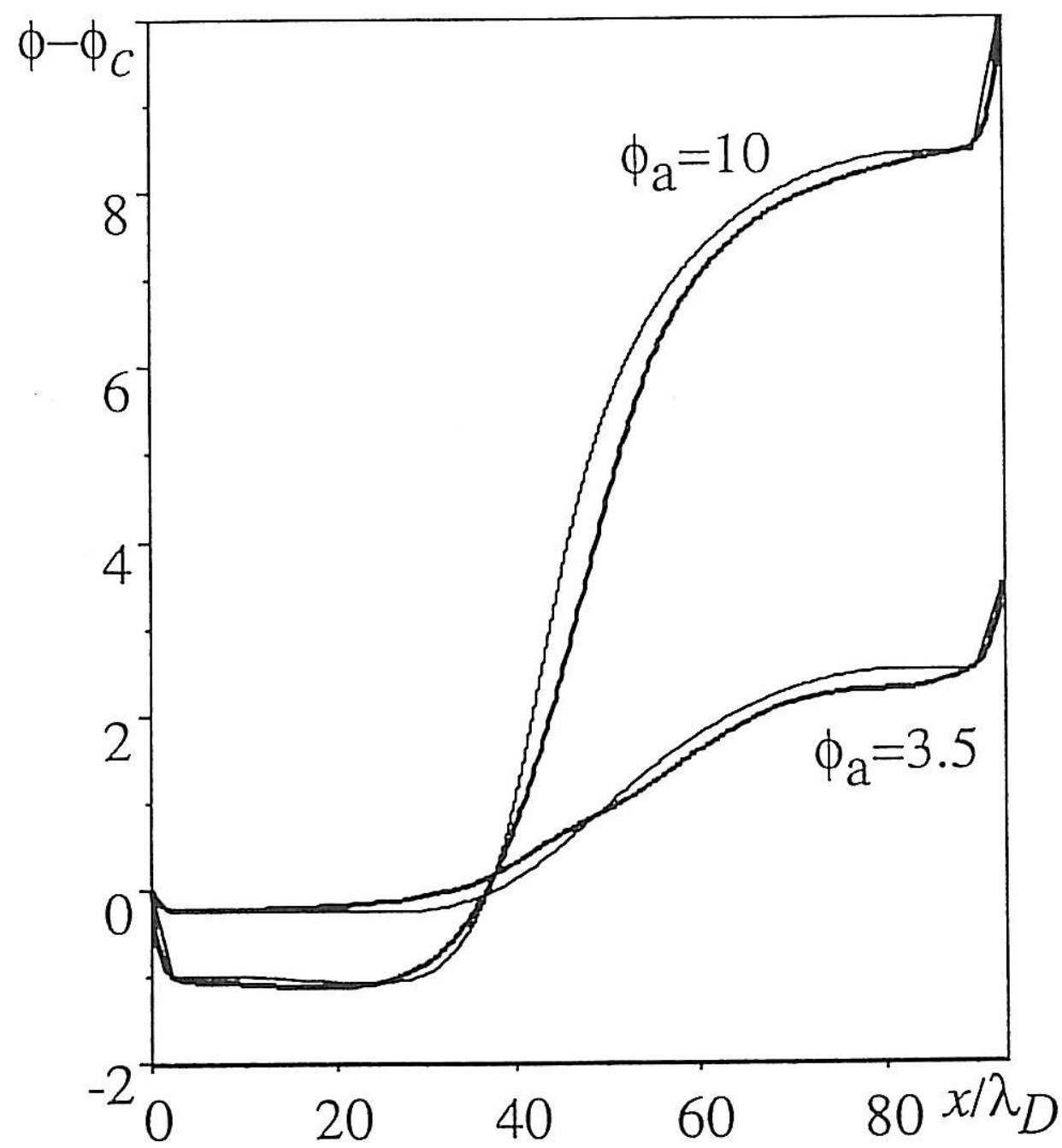

Fig. 3. Comparison between potential profiles evaluated theoretically (thin lines $)$ and profiles simulated (heavy lines) for $\phi_{a}=10\left(\Delta N / N_{1}=0.83\right.$, $\left.N_{0} / N_{1}=2.08\right)$ and $\phi_{a}=3.5 \quad\left(\Delta N / N_{1}=0.54, N_{0} / N_{1}=1.04\right)$. The profiles simulated remained constant for about $200 \mathrm{\omega}_{\mathrm{pe}}{ }^{-1}$ after the initial transient. In the theoretical profiles the sheath potential drops, evaluated by determining $\phi_{c}$ from (5), are indicated by straight lines in the sheath regions. 
TRITA-EPP-90-03

The Royal Institute of Technology, Department of Plasma Physics, S-10044 Stockholm Sweden

Potential Drops Supported by lon Density Cavities in the Dynamic Response of a Plasma Diode to an Applied Field

M. Bohm and S. Torvén

June 1990, 14 pages incl. ill., in English

Experiments have shown that an applied voltage drop may either be supported by a cathode sheath or by a quasi-linear variation over the plasma lasting for several electron transit times. In the latter case an ion density cavity existed initially. An analytical model and numerical simulations are used to show that a cavity gives rise to a quasi-linear potential variation for applied voltage drops below a certain critical value. For larger values the drop concentrates to a cathode sheath. The quasi-linear profile steepens to a double layer for large cavity depths.

Key words: Plasma Diode, Magnetic-Field-Aligned Electric Field, Ion Density Cavity, Electric Double Layer 